Concept Paper

\title{
A CSF Shunt System for Liquorpheresis and CSF Replacement
}

\author{
Manuel Menéndez González 1,2,3 \\ ${ }^{1}$ Servicio de Neurología, Hospital Universitario Central de Asturias, 33011 Oviedo, Asturias, Spain \\ 2 Departamento de Morfología y Biología Celular, Universidad de Oviedo, 33003 Oviedo, Asturias, Spain \\ ${ }^{3}$ Facultad de Ciencias de la Salud, Universidad Autónoma de Chile, Novena Región de La Araucanía, Chile
} menendezgmanuel@uniovi.es

\begin{abstract}
Liquorpheresis (CSF filtration) comprises a therapeutical approach that has been proposed to treat several neurological conditions where antibodies, inflammatory mediators or abnormal peptides are the cause or play an important role in the pathogenesis of the disease. Continuous or intermittent CSF replacement may be an alternative approach not explored so far.

Here, we review previous experiences in the use of liquorpheresis in autoimmune and degenerative neurological diseases. Then we describe a bidirectional CSF shunt system allowing portable liquorpheresis. Alternatively, CSF can be replaced with artificial cerebrospinal fluid. Both options would lead to mechanical dilution of the patient's CSF.
\end{abstract}

Keywords: csf; shunt; filtration; neurodegenerative; neuroimmunology; alzheimer; parkinson; guillain-barre; device; ventriculo-peritoneal shunt

\section{Introduction}

Liquorpheresis (CSF filtration) comprises a new therapeutical approach that has been proposed to treat several neurological conditions where soluble proteins or peptides within the nervous system play an important role in the physiopathology of the disease. This is the case of several autoimmune diseases where antibodies are involved affecting the central nervous system or the nerve roots. Among them, Guillain-Barrè syndrome (GBS) is perhaps the one with more evidence supporting its use. This disorder is usually treated with infusion of intravenous immunoglobulins. Corticoids, immunosuppressants and plasma exchange are alternatives. A clinical trial tested liquorpheresis in GBS. 
Although the number of patients was small, it was found that liquorpheresis is at least as effective as with plasma exchange [1, 2]. Multiple Sclerosis (MS), other autoimmune disorder, is usually treated with immunomodulatory and immunosuppressant drugs. Plasmapheresis was used with some success in open and controlled trials in some cases with acute or fulminant course of the disease. A probably better option than plasmapheresis would be liquorpheresis, since the autoimmune reaction is produced within the central nervous system. In a pilot-study where ten MS patients were treated with liquorpheresis, six patients improved in some extent. In all MS patients CSF-filtration was well tolerated, adverse events or complications were not observed [3]. Other autoimmune diseases where liquorpheresis was tried are single case reports and therefore provide limited evidence. For instance, in a case of lupus erythematosus with cerebral involvement liquorpheresis led to rapid and lasting neurological improvement and clinical cure [4].

The production and deposit of toxic peptides is a central fact in the physiopathology of all neurodegenerative diseases. The distinct protein aggregates that are found in neurodegenerative disorders and prion diseases seem to cause these disorders. Small intermediates (soluble oligomers) in the aggregation process can confer synaptic dysfunction, whereas large, insoluble deposits can cause cellular damage. Findings in other neurodegenerative diseases indicate that a broadly similar process of neuronal dysfunction is induced by diffusible oligomers of misfolded proteins [5]. Then, we propose that neurodegenerative diseases are also suitable to be treated by debugging CSF from those proteins. For instance Alzheimer's disease (AD) might be treated by removing beta-amyloid and phospho-Tau, Parkinson's disease (PD) and Lewy Bodies disease might be treated by removing alpha-synuclein, Huntington's disease (HD) by removing huntingtin protein and so on. Indeed, liquorpheresis has already been tried in some neurodegenerative diseases, such as in one patient with familial amyotrophic lateral sclerosis (ALS), where it did not show positive results [6].

We devise a bidirectional CSF shunt system allowing portable liquorpheresis. Alternatively, CSF can replaced with artificial cerebrospinal fluid (aCSF). Both options would lead to mechanical dilution of the patient's CSF. 


\section{Technical Report}

The following paragraphs have the objective of describing the setting and assembly of a CSF shunt system for liquorpheresis and CSF replacement.

\section{State of the art}

Standard ventricular-peritoneal shunting

Ventricular-peritoneal (or lumbar-peritoneal) shunting is the usual treatment of hydrocephalus, a condition produced when the natural system for draining and absorbing CSF does not work properly and the ventricles enlarge to accommodate the extra fluid. Systems for ventricular-peritoneal (or lumbar-peritoneal) shunting allow draining CSF from the ventricular system (or from the spinal subarachnoid space in the case of lumbar-peritoneal shunting) to the peritoneum. A shunt usually consists of two simple catheters (single light) and a one-way valve. The valve regulates the amount, flow direction and the intraventricular pressure that will cerebrospinal fluid out of the brain ventricles. As pressure increases within the brain cerebrospinal fluid, the check valve opens and excess liquid is drained to another body. There are various types of shunts. The two most common are: 1. Fixed pressure valves that regulate the amount of cerebrospinal fluid is drained based on a predetermined pressure setting. 2. Adjustable pressure valves that regulate the amount of cerebrospinal fluid that is drained based on a pressure value which can be adjusted. Using specially designed magnetic tools, neurosurgeons may change noninvasively the pressure level of the implanted adjustable valve during a visit in consultation, without another surgical procedure. Both types of valve may include an anti-siphon device control. The purpose of an anti-siphon device control is minimize overdrainage due to the force of gravity, which can cause more cerebrospinal fluid to drain when the individual is upright. In addition, some valves include a reservoir that can be used to check the operation of the shunt and to obtain samples of cerebrospinal fluid for laboratory studies.

\section{Bidirectional ventricular-peritoneal shunting}

Yang Qin and Jian W. Gu have proposed a surgical procedure ventricular-peritoneal shunting to improve the homeostasis of CSF, as a new treatment for AD, very recently [9]. 
It consists of a ventriculo-peritoneal shunt, an injection port and a portable infusion pump. Artificial CSF is pumped into the ventricles and the artificial CSF composition, infusion modes and pressure threshold of shunting can be adjusted according to the intracranial pressure and CSF contents.

\section{Description of a CSF shunt system for liquorpheresis and CSF replacement}

\section{General description}

Standard ventricular-peritoneal (or lumbar-peritoneal) shunting does not allow a replacement of cerebrospinal fluid as catheters are unidirectional. The system by Qin\&Gu allows bidirectional flow, but it is conceived just as a method to improve the homeostasis of CSF, not specifically for clearing .

Using these systems as starting point, we propose a new and simple improvement, where the main improvement would be a continuous pump, well infusing aCSF (CSF replacement) well the own patient's filtered CSF (liquorpheresis). This model comprises a series of interconnected elements, reservoirs, and access ports, allowing both to drain CSF to the outside (to an external collector) or to the peritoneum and to infuse CSF into the ventricular system (ventriculo-peritoneal) or the spinal subarachnoid space (lumboperitoneal shunt). Infusing CSF can be both aCSF (CSF replacement) and filtered CSF (liquorpheresis). Key elements in this model are the following (figures 1 and 2):

- Double lumen ventricular catheter (one inlet and one output), which allows both draining CSF from the ventricular system and infusing fluids. As an alternative to placement in the ventricular system (ventriculo-peritoneal shunt), the catheter can also be placed at the spinal level in the lumbar subarachnoid space (lumboperitoneal shunt).

- Double valve (bi-directional), which allows to regulate the pressure both input and output.

- Double lumen subcutaneous catheter (one inlet and one output, connected to homonyms ventricular catheters through the double valve), which like the ventricular catheter allows fluids to drain cerebrospinal fluid or infuse.

- A subcutaneous injection port connected to the gateway subcutaneous catheter, which allows fluid infusion by an external pump. Additionally it can also allow access for infusing drugs with syringe if needed. 
- External pump allowing continuous or intermittent infusion. It is connected to the injection port by an infusion apparatus with an L type non-coring needle.

\section{Versions}

This system may has two different versions intended for CSF replacement (version A) of CSF filtration (version B). Thus, the pump can be complemented with a deposit of aCSF (version $\mathrm{A}$, figure 1) or with a filter (version $\mathrm{B}$, figure 2 ).

In version $A$, the fluid to be infused is externally administered to the pump from a cartridge. The subcutaneous simple drainage catheter drains through an orifice into the peritoneal space (or outside). Thus, the proximal and the distal ends of the draining catheter are placed into the frontal horn of the lateral ventricle (or in the spinal subarachnoid space) and the peritoneal cavity (or to an external collector), respectively. aCSF has been first produced in 1949 and refined since then with the purpose of using it as a vehicle solution for administration of test agents to the central nervous system and neuroendoscopic surgery [7-8]. Today, with the availability of aCSF, CSF replacement seems a feasible intervention from a technical point of view. Up to our knowledge, continuous or intermittent CSF replacement has not been explored so far in the treatment of neurological conditions.

In version $B$, there is a LCR filter attached to the pump, which takes its source from the single subcutaneous drainage catheter. Thus, the proximal and the distal ends of the draining catheter are placed into the frontal horn of the lateral ventricle (or in the spinal subarachnoid space) and to the filter, respectively. Several CSF filters have already been developed and patented. 


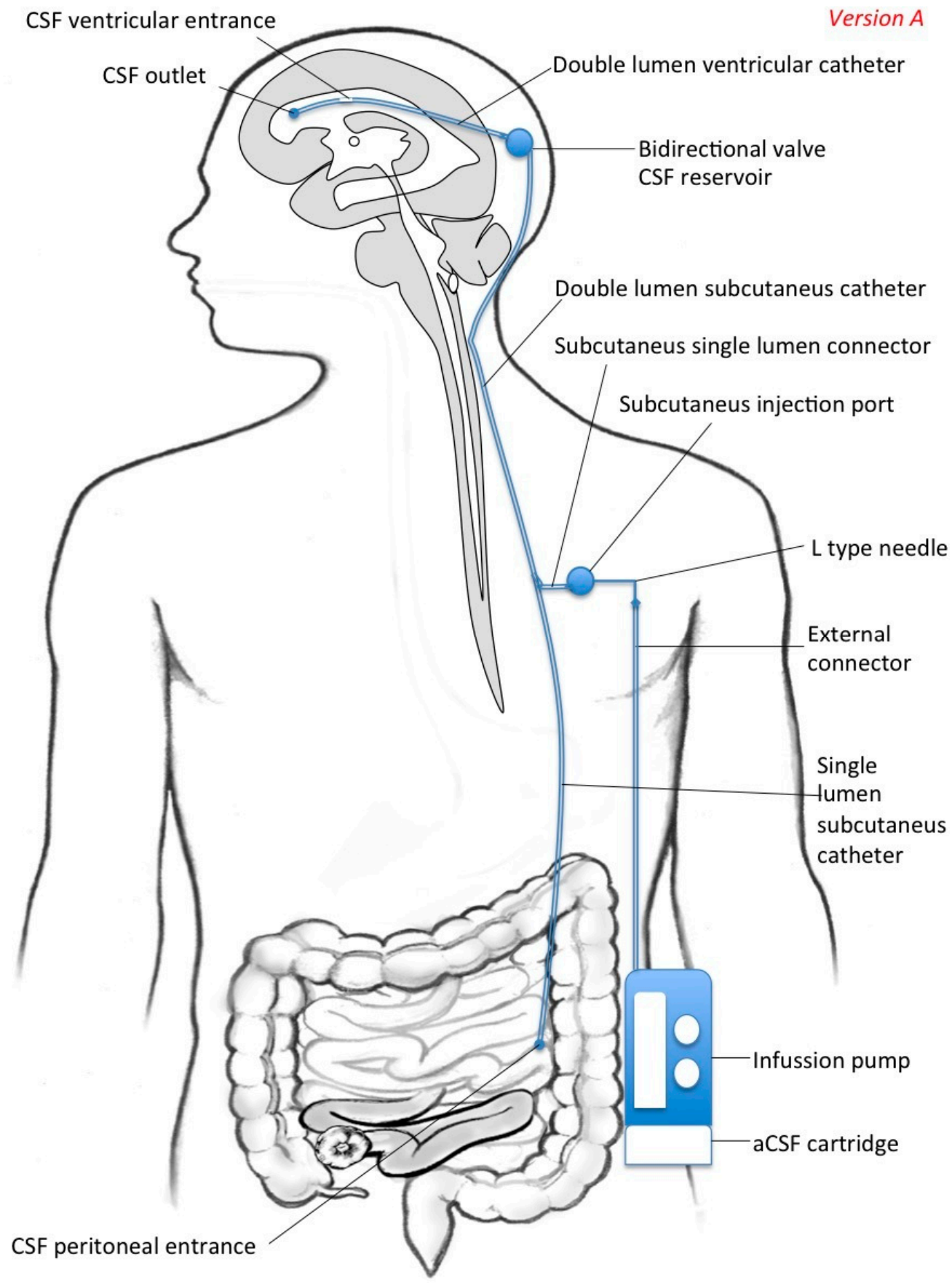

Figure 1: Version A. The fluid to be infused is externally administered to the pump with a cardridge. The subcutaneous simple drainage catheter drains through an orifice into the peritoneal space. 


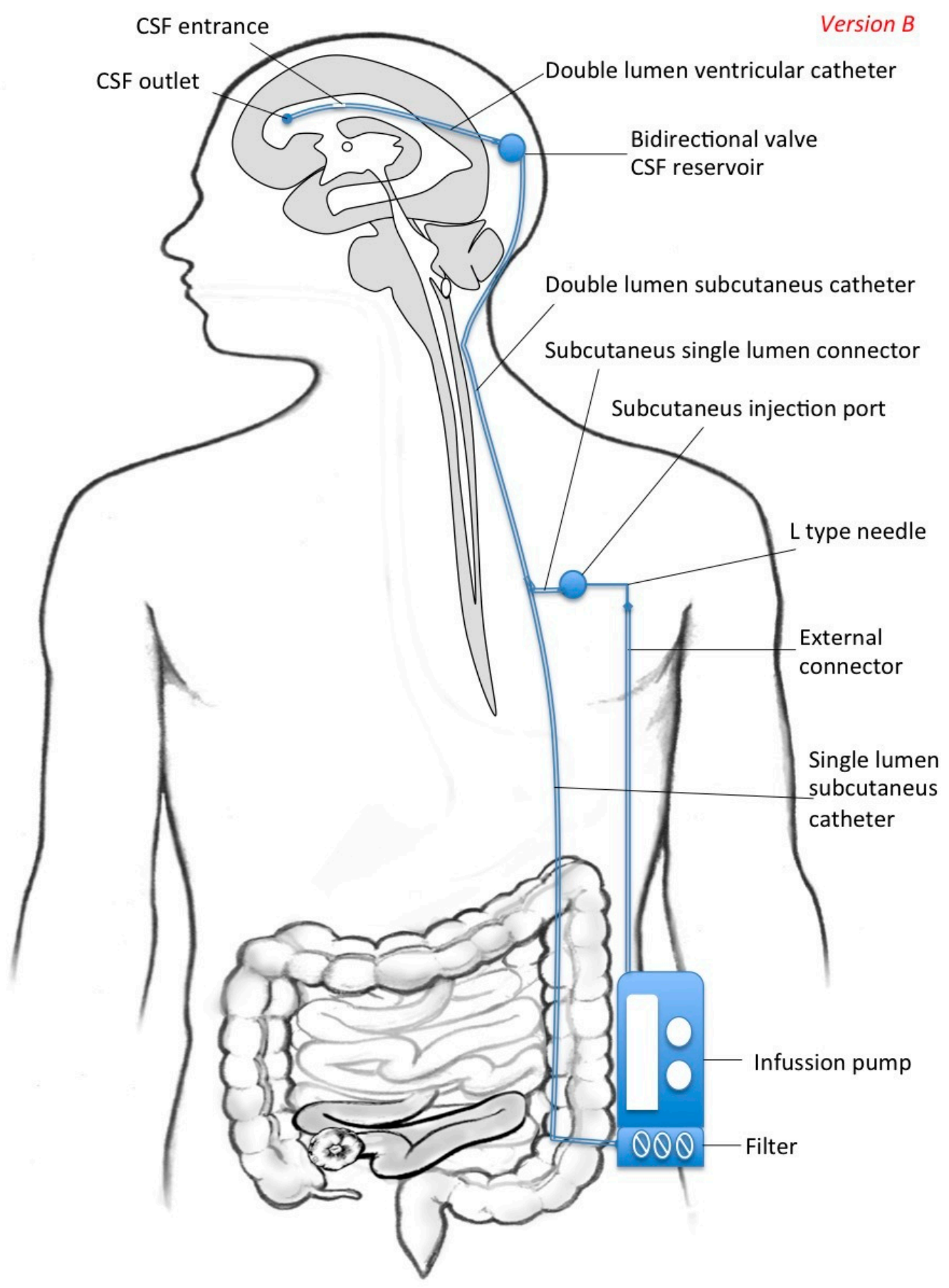

Figure 2: Version B. There is a LCR filter attached to the pump, which takes its source from the single subcutaneous drainage catheter. 
The innovative element in this model is that the pump can be complemented not only with a deposit of aCSF (as in the system by Qin\&Gu) but with a filter, allowing CSF replacement or liquorpheresis respectively. Both options ease the replacement of "dirty" CSF (with neurotoxic proteins) by "clean" CSF (without neurotoxic proteins) which can help fighting many neurological conditions.

Implanting this system requires a surgical procedure very similar to that used for the placement of valves ventriculo-peritoneal or lumbar-peritoneal shunt. In order to maximize the dilutional effect, the system may be programed to work in an alternate mode where the drainage and infusion of clean CSF is sequentially produced to avoid the new and "clean" CSF mixing with all the "dirty" CSF in the ventricular system that would happen if the system worked in a continuous in-out mode. Thus, some amount of "dirty" CSF should be drained before the infusion of "clean" CSF starts.

\section{Conclusions}

Liquorpheresis (CSF filtration) comprises a therapeutical approach that has been proposed to treat several neurological conditions. Developing wearable systems for liquorpheresis seems suitable today from a technical point of view. Also, continuous or intermittent replacement of CSF with aCSF is an alternative approach for treating these conditions that has not been explored so far.

We describe a bidirectional CSF shunt system that can be complemented either with a deposit of artificial CSF or with a CSF filter, allowing CSF replacement or liquorpheresis respectively. Both options would lead to mechanical dilution of the patient's CSF. 


\section{References}

1. Czosnyka M, Czosnyka Z, Momjian S, Pickard JD: Cerebrospinal fluid dynamics. Physiol Meas. 2004, 25(5):R51-76.

2. Wollinsky KH, Hülser PJ, Brinkmeier H, Aulkemeyer P, Bössenecker W, HuberHartmann KH, Rohrbach $\mathrm{P}$, Schreiber H, Weber F, Kron M, Büchele G, Mehrkens $\mathrm{HH}$, Ludolph AC, Rüdel R: CSF filtration is an effective treatment of Guillain-Barré syndrome: a randomized clinical trial. Neurology. 2001, 57(5):77480.

3. Haas J, Sailer M, Duzel E, Tendolkar I, Wurster U: CSF-filtration: An experimental therapeutical approach in multiple sclerosis treatment. Neuropsychiatrie. 1995, 9 (2):109-111.

4. Pfausler B, Bösch S, Grubwieser G, Vollert H, Greil R, Hagn C, Schmutzhard E: Multimodal therapy in life-threatening cerebral lupus erythematosus: the benefit of cerebrospinal fluid pheresis. Int Arch Allergy Immunol. 1995, 107(4):592-4.

5. Haass C, Selkoe DJ: Soluble protein oligomers in neurodegeneration: lessons from the Alzheimer's amyloid beta-peptide. Nat Rev Mol Cell Biol. 2007, 8(2):101-12.

6. Finsterer J, Mamoli B: Liquorpheresis (CSF filtration) in familial amyotrophic lateral sclerosis . Spinal Cord. 1999, 37(8):592-3.

7. Elliott KA, Lewis RC: Clinical uses of an artificial cerebrospinal fluid. Neurosurg. 1950, 7(3):256-60.

8. Shiobara R, Ohira T, Doi K, Nishimura M,Kawase T: Development of Artificial Cerebrospinal Fluid: Basic Experiments, and Phase Iland III Clinical Trials. Clinical Trials. J Neurol Neurophysiol . 2013, 4:173. 10.4172/2155-9562.1000173

9. Qin Y and Gu JW: A surgical method to improve the homeostasis of CSF for the treatment of Alzheimer's disease. Front. Aging Neurosci. 2016, 8:261. 10.3389/fnagi.2016.00261 